\section{Flicker electroretinograms recorded with mydriasis-free RETeval system before and after cataract surgery}

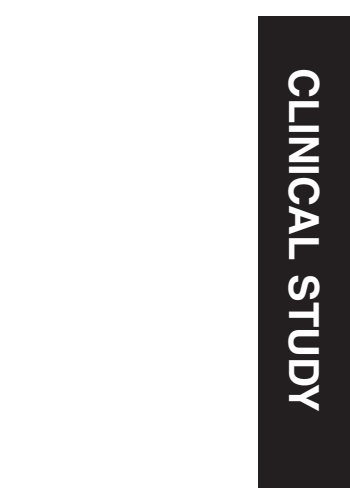

\begin{abstract}
Purpose The purpose of this study is to compare the amplitudes and peak times of the flicker electroretinograms (ERGs) recorded before and after cataract surgery with the RETeval system (LKC Technologies, Gaithersburg, MD, USA) from eyes without dilation.

Patients and methods Thirty-two eyes of 32 patients $(77.3 \pm 6.5$ years) that had grade 2 Emery-Little nuclear or cortical cataract without any other abnormalities were studied. Flicker ERGs were recorded with the RETeval system under mydriatic-free conditions. Skin electrodes were used to pick-up the ERGs that were elicited by white light delivered at a rate of $28.3 \mathrm{~Hz}$ and intensity of 2, 8, and $32 \mathrm{Td}$-s. The amplitudes and peak times of the flicker ERGs before and after cataract surgery were compared.
\end{abstract}

Results The mean amplitudes were significantly increased after the removal of the cataractous lenses at the three stimulus intensities (2 Td-s, $P<0.0001 ; 8$ Td-s, $P<0.0001$; and 32 Td-s, $P<0.0001)$. The mean peak times of the flicker ERGs elicited by 2 and 8 Td-s were significantly shortened after the surgery ( $2 \mathrm{td}-\mathrm{s}, P<0.0001$ and $8 \mathrm{td}-\mathrm{s}$, $P=0.0127)$ but not at $32 \mathrm{td}-\mathrm{s}(P=0.595)$.

Conclusions These results indicate that the presence of cataracts will affect both the amplitudes and the peak times of the flicker ERGs even if the cataract is mild. In addition, the results indicate that as stimulus intensity increases, the peak times is less affected by a cataract. The clarity of the crystalline lens should be considered when interpreting the flicker ERGs recorded with the RETeval system.

Eye (2017) 31, 1589-1593; doi:10.1038/eye.2017.110; published online 16 June 2017

G Miura, E Sato and S Yamamoto
Introduction

The RETeval system (LKC Technologies) is a noninvasive, mydriatic-free flicker electroretinographic (ERG) system that can elicit widely used to detect and assess the retinal ischemic damages in eyes with diabetic retinopathy and retinal vein occlusions. ${ }^{2-4} \mathrm{We}$ have reported that the amplitudes and peak times of the flicker ERGs recorded with the RETeval system at 8 Td-s were affected by cataracts. ${ }^{5}$ In that study, we examined the amplitudes and peak times of the flicker ERGs elicited by a stimulus intensity of $8 \mathrm{Td}$-s in eyes with Grade 2 and Grade 3 cataracts, and also from pseudophakic eyes. Our results showed that the mean amplitude was significantly smaller and the mean peak times was significantly longer in eyes with both grades of cataracts. However, the stimulus intensity, which is the recommended default setting for flicker ERGs for eyes without dilation in the RETeval system, is much weaker than the intensity recommended by International Society for Clinical Electrophysiology of Vision for conventional ERGs. ${ }^{6}$ In addition, the eyes were from different populations in the two cataract groups and also for the IOL group.

A cataract is one of the most frequent type of ophthalmologic disorder especially in the elderly, so it is important to know how much impact cataracts will have on the flicker ERGs elicited by different stimulus intensities and recorded with the RETeval system.

Thus, the purpose of this study was to determine the effect of a cataract on the RETeval flicker ERGs elicited by different stimulus intensities and recorded from the same eye before and after cataract surgery. and record flicker ERGs. ${ }^{1}$ The RETeval system is
Department of Ophthalmology and Visual Science, Chiba University Graduate School of Medicine, Chiba, Japan

Correspondence: G Miura, Department of Ophthalmology and Visual Science, Chiba University Graduate School of Medicine, Inohana 1-8-1, Chuo-ku, Chiba 260-8670, Japan Fax: +8143224 4162 E-mail: gmiura2@chiba-u.jp

Received: 3 August 2016 Accepted in revised form: 9 May 2017 Published online: 16 June 2017
Tel: +81 43226 2124; 


\section{Materials and methods}

Thirty-two eyes of 32 patients that had undergone cataract surgery at the Chiba University Hospital between June 2015 and June 2016 were studied. All of the patients had had mydriasis-free flicker ERGs recorded before and after the surgery. The procedures used in this study conformed to the tenets of the Declaration of Helsinki and were approved by the Institutional Review Board of Chiba University Hospital (number 2075). An informed consent was obtained from each patient for the surgery and for the ERG recordings.

All patients had a complete ophthalmic examination including measurements of the best-corrected visual acuity (BCVA) and intraocular pressure. In addition, all had slitlamp examinations and indirect ophthalmoscopy. The BCVA was measured monocularly using a Japanese standard Landolt ring chart (System Charts SC-2000 Nidek Instruments, Gamagori, Japan) at a distance of $5 \mathrm{~m}$. The decimal visual acuities were converted to the logarithm of the minimum angle of resolution (logMAR) units for the statistical analyses. Only subjects with Grade 2 cataract based on the Emery-Little classification ${ }^{7}$ were studied.

None of the patients had any other ocular abnormalities in the anterior segment, media, and fundus, and all eyes had phacoemulsification and intraocular lens implantation surgery through a superior sclera-corneal incision without any complications. Yellow-colored acrylic foldable intraocular lenses were implanted in all of the eyes. None of the patients had any surgery-related complications. Eyes with refractive errors (spherical equivalents) greater than \pm 6 diopters and patients with diabetes mellitus were excluded.

Flicker ERGs were recorded with the RETeval system (LKC Technologies) from all patients under mydriatic-free conditions before and after the cataract surgery. The pupil size of all of the patients ranged from 2 to $4 \mathrm{~mm}$ during the ERG recordings. Skin electrodes were used to pick-up the ERGs that were elicited by white light delivered at a frequency of $28.3 \mathrm{~Hz}$ and intensity of 2, 8, and $32 \mathrm{Td}-\mathrm{s}$. No background light was used. The contralateral eye was covered during the recordings. The amplitudes and peak times of the fundamental component were automatically extracted and displayed by the RETeval system using an embedded algorithm.

Wilcoxon signed-rank tests were used to determine the significance of the differences in the amplitudes and peak times of the flicker ERGs. A $P$-value of $<0.05$ was taken to be statistically significant.

\section{Results}

We studied 32 eyes of 32 patients (12 men and 20 women) whose mean age was $77.3 \pm 6.5$ years with a range of 64 to
90 years. The mean BCVA was $0.51 \pm 0.38 \log$ MAR units with a range of 1.30 to $0.04 \log \mathrm{MAR}$ units before the cataract surgery and $0.004 \pm 0.08 \log$ MAR units with a range of 0.10 to $-0.08 \log$ MAR units after the surgery (Table 1). The BCVA was significantly improved after the cataract surgery $(P<0.0001)$.

The mean amplitude and peak times before surgery were $2.7 \pm 1.3 \mu \mathrm{V}$ and $40.7 \pm 2.2 \mathrm{~ms}$ with $2 \mathrm{Td}-\mathrm{s}$, $7.8 \pm 2.9 \mu \mathrm{V}$ and $35.4 \pm 2.3 \mathrm{~ms}$ with $8 \mathrm{Td}-\mathrm{s}$, and $12.6 \pm 4.3 \mu \mathrm{V}$ and $31.4 \pm 2.5 \mathrm{~ms}$ with $32 \mathrm{Td}-\mathrm{s}$. The mean amplitude and peak times after surgery were $4.5 \pm 2.1 \mu \mathrm{V}$ and $39.5 \pm 2.2 \mathrm{~ms}$ with $2 \mathrm{Td}-\mathrm{s}, 12.3 \pm 4.9 \mu \mathrm{V}$ and $35.0 \pm 2.1 \mathrm{~ms}$ with $8 \mathrm{Td}-\mathrm{s}$, and $19.0 \pm 5.5 \mu \mathrm{V}$ and $31.3 \pm 2.2 \mathrm{~ms}$ with $32 \mathrm{Td}-\mathrm{s}$ (Tables 2 and 3 ). The mean amplitudes were significantly increased after the removal of the cataractous lens for all stimulus intensities $(P<0.0001,2$ Td-s; $P<0.0001,8$ Td-s; and $P<0.0001,32$ Td-s). The mean peak times for intensities of 2 and 8 Td-s were significantly shortened after the surgery ( $2 \mathrm{td}-\mathrm{s}$, $P<0.0001$ and $8 \mathrm{td}-\mathrm{s}, P=0.0127)$, but not for $32 \mathrm{Td}-\mathrm{s}$. The mean peak times for the flicker ERGs elicited by $32 \mathrm{Td}-\mathrm{s}$ was not significantly different after the cataract surgery $(P=0.595)$.

Eighteen eyes had nuclear cataracts and 14 eyes had cortical cataracts. The mean amplitude of the flicker ERGs before surgery was $2.9 \pm 1.4 \mu \mathrm{V}$ with $2 \mathrm{Td}-\mathrm{s}, 8.0 \pm 3.2 \mu \mathrm{V}$ with $8 \mathrm{Td}$-s, and $12.2 \pm 4.7 \mu \mathrm{V}$ with $32 \mathrm{Td}$-s for eyes with nuclear cataract. The mean amplitude of the flicker ERGs before surgery was $2.5 \pm 1.0 \mu \mathrm{V}$ with $2 \mathrm{Td}-\mathrm{s}, 7.4 \pm 2.4 \mu \mathrm{V}$ with $8 \mathrm{Td}$-s, and $13.0 \pm 3.7 \mu \mathrm{V}$ with $32 \mathrm{Td}$-s for eyes with cortical cataract. There was no significant difference between the amplitudes for both types of cataracts before

Table 1 Pre- and postoperative demographics of the patients studied

\begin{tabular}{lc}
\hline Number of eyes & 32 \\
Age (years) & $77.3 \pm 6.5$ \\
Sex (male/female) & $12 / 20$ \\
LogMAR VA before surgery & $0.51 \pm 0.38$ \\
LogMAR VA after surgery & $0.004 \pm 0.08$ \\
Types of cataract (cortical/nuclear) & $14 / 18$
\end{tabular}

Abbreviation: LogMAR, logarithm of the minimum angle of resolution. Data are expressed as mean \pm SD.

Table 2 Amplitudes of flicker ERGs recorded before and after cataract surgery elicited by a stimulus intensity of 2,8 , or $32 \mathrm{Td}$-s

\begin{tabular}{lccc}
\hline Intensity $(\mathrm{Td}$-s) & Before surgery $(\mu v)$ & After surgery $(\mu v)$ & P-value \\
\hline 2 & $2.7 \pm 1.3$ & $4.5 \pm 2.1$ & $<0.0001$ \\
8 & $7.8 \pm 2.9$ & $12.3 \pm 4.9$ & $<0.0001$ \\
32 & $12.6 \pm 4.3$ & $19.0 \pm 5.5$ & $<0.0001$ \\
\hline
\end{tabular}


surgery $(P=0.38,2$ Td-s; $P=0.50,8$ Td-s; and $P=0.62,32$ Td-s). The average peak times of the flicker ERGs before surgery was $40.0 \pm 1.5 \mathrm{~ms}$ with $2 \mathrm{Td}-\mathrm{s}, 35.2 \pm 1.6 \mathrm{~ms}$ with 8 Td-s, and $31.1 \pm 1.8 \mathrm{~ms}$ with $32 \mathrm{Td}$-s for eyes with nuclear cataract. The average peak times of the flicker ERGs before surgery was $41.4 \pm 2.8 \mathrm{~ms}$ with $2 \mathrm{Td}-\mathrm{s}, 35.6 \pm 3.0 \mathrm{~ms}$ with $8 \mathrm{Td}$-s, and $31.8 \pm 3.3 \mathrm{~ms}$ with $32 \mathrm{Td}$-s for eyes with cortical cataracts. There was no significant difference between the peak times for both types of cataracts $(P=0.15,2$ Td-s; $P=0.62,8$ Td-s; and $P=0.43,32$ Td-s $)$ (Figure 1).

Table 3 Peak times of flicker ERGs recorded before and after cataract surgery elicited by intensity of 2,8 , or $32 \mathrm{Td}-\mathrm{s}$

\begin{tabular}{lccc}
\hline Intensity (Td-s) & Before surgery (ms) & After surgery (ms) & P-value \\
\hline 2 & $40.7 \pm 2.2$ & $39.5 \pm 2.2$ & $<0.0001$ \\
8 & $35.4 \pm 2.3$ & $34.9 \pm 2.2$ & 0.0127 \\
32 & $31.4 \pm 2.5$ & $31.3 \pm 2.2$ & 0.595 \\
\hline
\end{tabular}

a

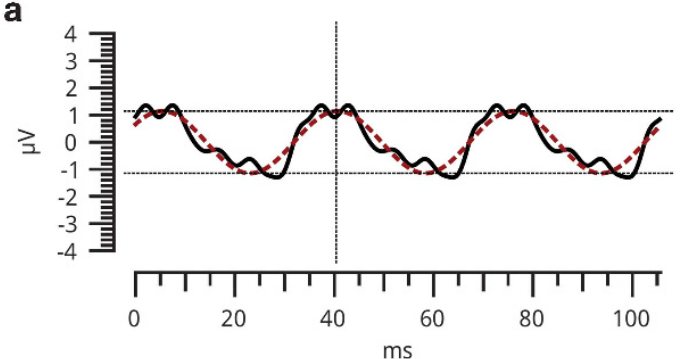

b

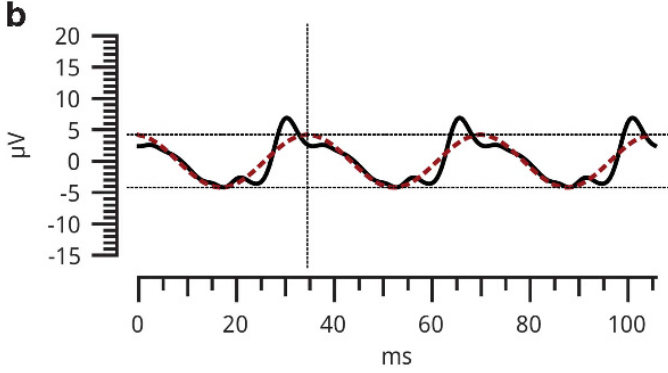

c

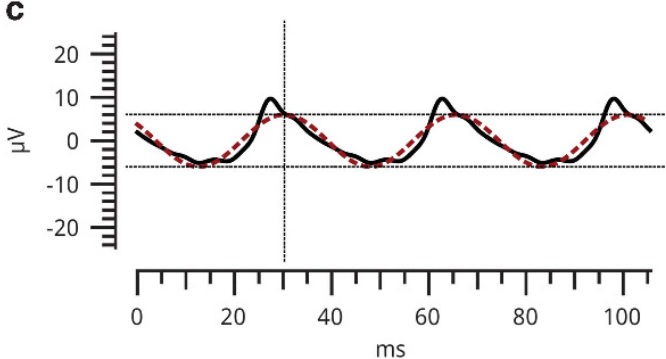

\section{Discussion}

Our results showed that Grade 2 cataracts will affect both the amplitudes and peak times of the flicker ERGs elicited by stimulus intensities of $<8 \mathrm{Td}$-s. Although the subjects in this study were different from those used in our earlier study, the results were quite similar. ${ }^{5}$

The new and important finding in this study was that the lens opacities can increase the peak times probably by weakening the intensity of the light stimulus, but the effect will be essentially eliminated when the stimulus intensity was equal to $32 \mathrm{Td}$-s. This study was performed only on eyes with Grade 2 Emery and Little cataracts, which are described as being 'semisoft: yellowish white,' which means that they were comparatively mild lens opacities. If cataracts denser than Grade 2 are present, the effect of the lens opacity on the flicker ERGs would be expected to be greater than the results of this study. These findings indicate that when testing for mild retinal ischemia, it is necessary to take into account the effects of cataracts.
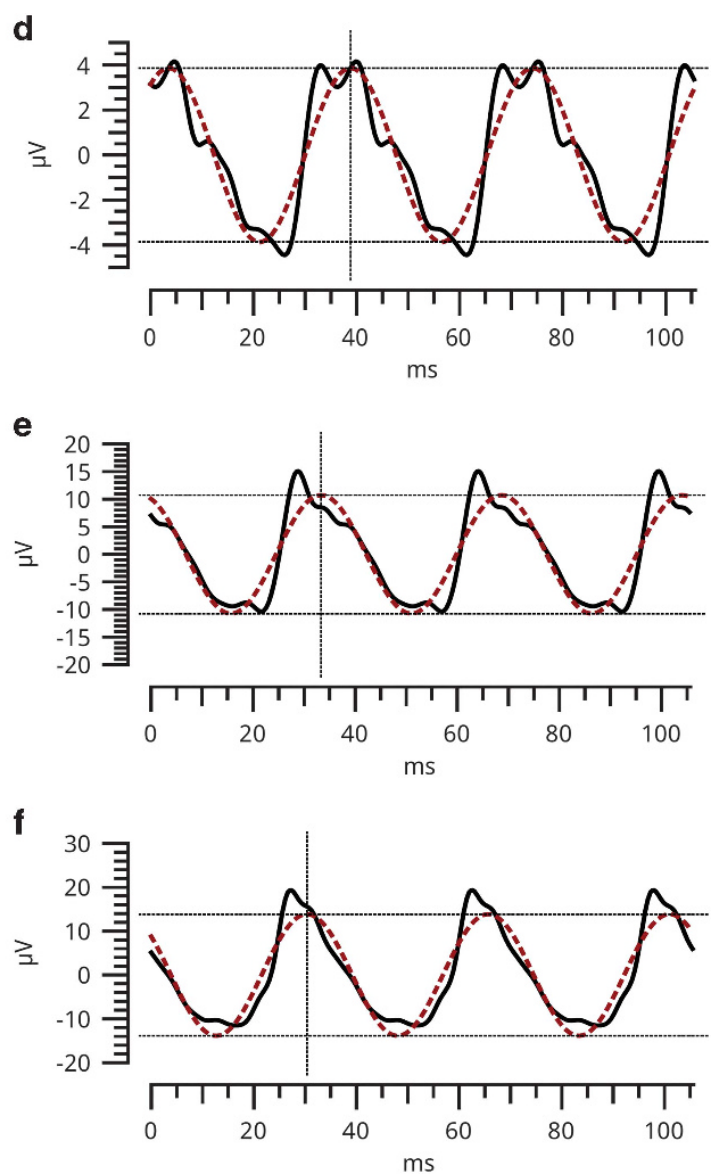

Figure 1 Representative flicker ERGs recorded with the RETeval system elicited intensity of $2(a, d), 8(b, e)$ and 32 (c, f) Td-s before (a, b, c) and after $(\mathrm{d}, \mathrm{e}, \mathrm{f})$ cataract surgery. Dotted lines are the superimposed fundamental component extracted by the RETeval system. Black lines are the actual waveforms. 
We studied eyes with nuclear cataracts and cortical cataracts. Earlier studies reported that the density of multifocal ERGs were affected different in the different areas of the area of the retina because of the light scattering by a cataract. 8,9 De waad et al ${ }^{10}$ reported that the different types of cataract display different light scatter characteristics.

Thus, to confirm whether there is a difference in the influence of the scattering of the light depending on the type of cataract, we investigated the differences between the amplitudes and the peak times of each type of cataract. Our results showed that there was no significant difference in the amplitudes and peak times between the two types of cataracts. These results indicate that the type of cataract most likely has no effect on the flicker ERGs in the mydriasis-free conditions when recordings are made with the RETeval system.

Kato et al ${ }^{1}$ showed that the peak times of the RETeval flicker ERGs was significantly prolonged when the pupil was larger than $\sim 6.5 \mathrm{~mm}$. The pupil sizes of all of our subjects ranged from 2 to $4 \mathrm{~mm}$ during the ERG recordings. Thus, our results should not be affected by the pupil diameter although the pupil size should be monitored and the peak times need to be analyzed carefully when examining the RETeval flicker ERGs.

There are some limitations in this study. The first limitation was that all of the patients were examined under mydriatic-free conditions. Thus, it is not entirely known how the lens opacities affect the flicker ERGs if the pupil diameter is different and the distribution of the light stimulus on the entire retina different. Thus, the impact of the cataract type and the degree of light scattering may also be different.

The second limitation was that only flicker ERGs were investigated. It is not known how lens opacities affect the other ERG components, especially the rod system because the flicker ERG mainly originated from the neural activity of the cone system. ${ }^{11}$ As the location of the cones in the retina is different from that of rods, it is estimated that the effect of lens opacities on the rod ERGs will be different.

The other limitation was that we did not compare waveforms of RETeval system and those recorded with the conventional ERG system. There have been several reports about the effects of cataracts on the amplitudes of conventional ERGs and densities of multifocal ERGs.9,12 They reported that amplitudes of conventional ERGs and densities were significantly decreased in eyes with a cataract. These results are consistent with ours. In addition, Yasuda et al ${ }^{13}$ reported that both the peak times and amplitudes recorded by the RETeval system and conventional system were significantly correlated. Thus, similar results may be obtained also by the conventional ERG system. Further investigations are needed.
In conclusion, the presence of Grade 2 cataracts affected both of the amplitudes and the peak times of the flicker ERGs, however the effect of lens opacity on the peak times decreased as strengthen the light intensity increased. The presence of lens opacities should be taken into consideration when interpreting the flicker ERGs recorded with the RETeval system even if the cataract is a relatively mild.

\section{Summary}

What was known before

- The RETeval system is widely used to detect and assess the retinal ischemic damages in eyes with diabetic retinopathy and retinal vein occlusions.

- Amplitudes and peak times of the flicker ERGs recorded with the RETeval system at 8 Td-s were affected by cataracts.

- The mean amplitude was significantly smaller and the mean peak time was significantly longer in eyes with cataracts.

What this study adds

- The effect of lens opacity on the peak time decreased as strengthen the light intensity increased. The effect will be essentially eliminated when the stimulus intensity was equal to 32 Td-s.

- There was no significant difference in the amplitudes and peak times between nuclear and cortical cataracts.

\section{Conflict of interest}

The authors declare no conflict of interest.

\section{Acknowledgements}

We thank Professor Duco Hamasaki, Bascom Palmer Eye Institute, University of Miami, for his critical discussion and editing of the final manuscript.

\section{References}

1 Kato K, Kondo M, Sugimoto M, Ikesugi K, Matsubara H. Effect of pupil size on flicker ERGs recorded with RETeval system: new mydriasis-free full-field ERG system. Invest Ophthalmol Vis Sci 2015; 56: 3684-3690.

2 Kjeka O, Bredrup C, Krohn J. Photopic $30 \mathrm{~Hz}$ flicker electroretinography predicts ocular neovascularization in central retinal vein occlusion. Acta Ophthalmol Scand 2007; 85: 640-643.

3 Larsson J, Bauer B, Andre'asson S. The 30-Hz flicker cone ERG for monitoring the early course of central retinal vein occlusion. Doc Ophthalmol 2008; 117(2): 103-109.

4 Holm K, Schroeder M, Lövestam AM. Peripheral retinal function assessed with $30-\mathrm{Hz}$ flicker seems to improve after treatment with Lucentis in patients with diabetic macular oedema. Doc Ophthalmol 2015; 131(1): 43-51.

5 Miura G, Nakamura Y, Sato E, Yamamoto S. Effects of cataracts on flicker electroretinograms recorded with 
RETeval system: new mydriasis-free ERG device. BMC Ophthalmol 2016; 16: 22.

6 McCulloch DL, Marmor MF, Brigell MG, Hamilton R, Holder GE, Tzekov R et al. ISCEV standard for full-field clinical electroretinography (2015 update). Doc Ophthalmol 2015; 130: 1-12.

7 Emery JM, Little JH (eds). Surgical techniques, complications and results. Phacoemulsification and Aspiration of Cataract. CV Mosby: St Louis, MO, USA, 1979, pp 45-48.

8 Chan HL, Siu AW, Yap MK, Brown B. The effect of light scattering on multifocal electroretinography. Ophthalmic Physiol Opt 2002; 22: 482-490.

9 Tam A, Chan H, Brown B, Yap M. The effects of forward light scattering on the multifocal electroretinogram. Curr Eye Res 2004; 28: 63-72.
10 de Waard PW, IJspeert JK, van den Berg TJ, de Jong PT. Intraocular light scattering in age-related cataracts. Invest Ophthalmol Vis Sci 1992; 33: 618-625.

11 Kondo M, Sieving PA. Primate photopic sine-wave flicker ERG: vector modeling analysis of component origins using glutamate analogs. Invest Ophthalmol Vis Sci 2001; 42: 305-312.

12 Cruz RD, Adachi-Usami E. Quantitative evaluation of electroretinogram before cataract surgery. Jpn J Ophthalmol 1989; 33: 451-457.

13 Yasuda S, Kachi S, Ueno S, Piao CH, Terasaki H. Flicker electroretinograms before and after intravitreal ranibizumab injection in eyes with central retinal vein occlusion. Acta Ophthalmol 2015; 93: e465-e468. 